ARTICLE HISTORY: Received: November 14, 2021 Accepted: January 6, 2022 Published: January 13, 2022

ПРОБЛЕМЫ ОЦЕНКИ НАДЕЖНОСТИ СЛОЖНЫХ ТЕХНИЧЕСКИХ СИСТЕМ

\author{
Плотников Григорий Александрович \\ Ассистент кафедры информационных технологий предпринимательства \\ Санкт-Петербургского государственного университета аэрокосмического приборостроения \\ 190000, РФ, Санкт-Петербург, ул. Большая Морская, д. 67, лит. А., ГУАП. \\ Скворода Дмитрий Владимирович \\ Ассистент кафедры информационных технологий предпринимательства \\ Санкт-Петербургского государственного университета аэрокосмического приборостроения \\ 190000, РФ, Санкт-Петербург, ул. Большая Морская, д. 67, лит. А., ГУАП.
}

\title{
PROBLEMS OF RELIABILITY ASSESSMENT OF COMPLEX TECHNICAL SYSTEMS
}

\author{
Plotnikov Grigory Alexandrovich \\ Assistant of the Department of Information Technology Entrepreneurship \\ Saint Petersburg State University of Aerospace Instrumentation \\ 190000, Russian Federation, Saint Petersburg, Bolshaya Morskaya str., 67/A. \\ Skvoroda Dmitry Vladimirovich \\ Assistant of the Department of Information Technology Entrepreneurship \\ Saint Petersburg State University of Aerospace Instrumentation \\ 190000, Russian Federation, Saint Petersburg, Bolshaya Morskaya str., 67/A.
}

\begin{abstract}
Аннотация. В статье рассматриваются проблемы и факторы, которые мешают произвести точную оценку надежности и безотказности функционирования сложных технических систем. Поднимаются вопросы моделирования и симуляции сложно - структурных объектов и систем.

Abstract. The article discusses the problems and factors that prevent an accurate assessment of the reliability and reliability of the functioning of complex technical systems. The issues of modeling and simulation of complex structural objects and systems are raised.
\end{abstract}

Ключевые слова: надежность, безотказность, улучшение качества, сложные системы.

Keywords: reliability, trouble-free, quality improvement, complex systems.

В настоящее время регулярно появляются новые технические и программные решения, которые позволяют улучшить жизнь как человека в отдельности, так и общества в целом. К сожалению, большая часть нововведений неизбежно приводит к большим финансовым и временным затратам. Чтобы минимизировать такие затраты и обеспечить наилучшее качество итогового продукта производится оценка надежности и безотказности технических продуктов. Оценка этих основополагающих показателей может производится двумя путями. Первый - математические вычисления с использованием законов распределения случайной величины [1]. Второй - построение готовой модели, которая подвергается ряду заранее разработанных тестовых симуляций.

Математическая оценка надежности и безотказности довольно быстрый и дешевый способ, чтобы рассчитать теоретическую вероятность работоспособности оборудования. Однако, точность результатов будет достаточно высокой только тогда, когда известна вероятность выхода из строя каждого компонента системы. Даже если отбросить элементы, которые не влияют на работу всей системы в целом, то останется десяток важных компонентов, таких как центральный процессор, материнская плата, оперативное (ОЗУ) и постоянное (ПЗУ) запоминающее устройство и многое другое. К сожалению, с точностью утверждать о числовом выражении надежности каждого из перечисленных компонентов даже не может идти речи. Даже если рассмотреть центральный процессор (ЦП), то возникнет ситуация полной неопределенности. Связано это с тем, что невозможно найти достоверные данные о проценте брака или среднюю безотказную продолжительность работы того или иного ЦП. Цифры по браку, которые предоставляются производителями несут больше маркетинговую ценность перед инвесторами, нежели важную информацию для покупателей. Также все показателя берутся от завода изготовителя и официальных дистрибьютеров в нескольких странах. По факту, проблема может иметь более глобальный характер. Хорошим примером служит история с процессорами AMD, в которых был обнаружен повсеместный брак и полная неработоспособность итогового продукта [2]. Таким образом, даже надежность одного из компонентов может сильно варьироваться, что в современных реалиях не позволяет довериться данной методике определения надежности сложной технической системы. 
В дополнение ко всему вышесказанному хочется отметить, что с большей долей вероятности мы можем утверждать о надежности и безотказности системы только при ее полном моделировании на компонентном уровне [3]. Когда есть полная модель, можно предусмотреть все конечные состояния такой системы и проанализировать все варианты развития событий. Однако, данная методика легко применяется только к устройствам и системам, у которой небольшой объем ПЗУ и ОЗУ, а лучше их полное отсутствие. В современных сложных системах объем памяти измеряется, в лучшем случае, гигабайтами. Каждый байт памяти, так или иначе, должен быть рассмотрен в готовой модели. Даже если у нас 1 ГБ ОЗУ и ПЗУ, это тысячи новых конечных состояний. Разумеется, сейчас реализовать данную задачу практически невозможно.

Исходя из проблем с математическим выражением надежности каждого компонента, единственным верным способом оценки надежности готовой системы кажется постройка готовой функциональной модели и ее дальнейшие испытания. Однако, стоимость итогового продукта на ранних этанах может достигать чрезвычайно больших значений. Также не стоит забывать и про временные затраты при проектировании и разработке такого продукта. Исходя из этого, можно прийти к выводу, что данный метод также не всегда подходит для решения задачи определения надежности и безотказности. Даже если будет обнаружено, что один из компонентов системы имеет низкую надежность, то подбор нового компонента может занять продолжительное время, а перепроектирование готового образца - очередная строка расходов и больших потерь.

Использование частичного или полного дублирование компонентов системы [4] также малоприменимы в современных реалиях. Сложно представить, что в современный дрон будет установлено по два процессора, пара материнских плат и других компонентов. От этого вес конечного устройства в значительной степени возрастет, что неблагоприятно скажется на длительности полета и выполнении поставленной перед ним миссии. Подобные нюансы и тонкости можно обнаружить при дублировании компонентов практически для каждой сложной системы. В одном случае, такое решение неблагоприятно скажется на потребительских свойствах продукта, в другом на итоговой стоимости.

Таким образом, одним из решений возникшей ситуации может стать разработка компьютерных программ симуляции работы сложных технических систем. Понятно, что в каждом конкретном случае придется вносить правки и корректировки, чтобы приблизить конечный продукт к реальным условиям. Тем не менее, построить такую программу можно будет на едином ядре с определенной логикой работы. Скорее всего, такие программы будут базироваться на квинтэссенции математического метода оценки надежности и реальной разработки модели готовой сложной системы. Разумеется, на проектирование такой системы и ее наполнение может занять большое количество времени. Тем не менее, это позволит совершить качественный рывок в вопросе оценки безотказности и надежности сложной технической системы.

\section{Список литературы:}

1. А.С. Ширшиков. Оценка надежности технических систем: учеб. пособие / А.С. Ширшиков, В.В. Лянденбурский, А.М. Белоковыльский. - Пенза: ПГУАС, 2015. - 240 с.

2. Сайт CNews (2022). Дата обращения 08.01.2022 https://www.cnews.ru/news/top/2021-0215 amd zavalila rynok brakovannymi

3. Сайт Habr (2022). Дата обращения 08.01.2022 https://habr.com/ru/post/307090/

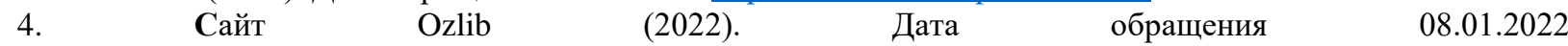

https://ozlib.com/984035/tehnika/rezervirovanie_nadezhnost_dublirovannoy_sistemy

\section{References:}

1. A.S. Shirshikov. Assessment of the reliability of technical systems: studies. manual / A.S. Shirshikov, V.V. Lyandenbursky, A.M. Belokylsky. - Penza: PGUAS, 2015. - 240 p.

2. CNews website (2022). Accessed $\mathbf{0 8 . 0 1 . 2 0 2 2}$ https://www.cnews.ru/news/top/2021-0215 amd zavalila rynok brakovannymi

3. Habr website (2022). Accessed 08.01.2022 https://habr.com/ru/post/307090/

4. Ozlib website (2022). Accessed

https://ozlib.com/984035/tehnika/rezervirovanie_nadezhnost_dublirovannoy_sistemy 